\title{
Moiré pattern induced by the electronic coupling between 1-Octanol self-assembled monolayer and graphite surface
}

\author{
Fabien Silly \\ CEA, IRAMIS, SPCSI, Hybrid Magnetic Nanoarchitectures, F-91191 Gif sur Yvette, France. \\ E-mail: fabien.silly@cea.fr
}

\begin{abstract}
Two-dimensional self-assembly of 1-Octanol molecules on graphite surface is investigated using scanning tunneling microscopy (STM) at the solid/liquid interface. STM images reveal that this molecule self-assembles into a compact hydrogen-bonded herringbone nanoarchitecture. Molecules are preferentially arranged in a head-to-head and tail-to-tail fashion. A Moiré pattern appears in the STM images when the 1-Octanol layer is covering the graphite surface. The large Moiré stripes are perpendicular to the 1-Octanol lamellae. Interpretation of the STM images suggests that the Moire periodicity is governed by the electronic properties of the graphite surface and the 1-Octanol layer periodicity.
\end{abstract}




\section{Introduction}

Engineering sophisticated organic nanostructures on surfaces is the focus of recent research interest [1, 2, 3]. Controlling molecular self-assembly offers unique directions for the fabrication of two-dimensional (2D) supramolecular nanoarchitectures. The resulting structures can be tailored at the nanometer scale by exploiting molecule-substrate $[4,5]$ or intermolecular interactions $[1,2]$. Molecular shape, size, structure and substituents are key parameters driving the structure of self-assembled nanoarchitectures. Alkane molecule is a particularly attractive building block because its extremity can easily be functionnalized to interact with other molecules or with the surface [6]. Numerous organic patterns resulting from modified-alkane self-assembled structures have been reported or predicted on graphite, graphene and other surfaces $[7,8,9,10]$.

Different lamellar structures have been experimentally observed depending of the molecule arrangement and configuration. The axis of the molecule forming the lamellae can be aligned $[11,12]$ or rotated by an angle [13] that is usually governed by substrate geometry. It has also been observed that molecules can lay flat or perpendicular to the surface $[10,7]$. Molecules can also adopt a straight or twisted configuration (cis or trans conformation) [14]. Racemic and enantiomer nanoarchitectures can be formed.

These structures can be exploited in nanotechnology as nanotemplate or active layer. For example substituted alkane derivative monolayer on graphene/ $\mathrm{SiO}_{2}$ has been successfully used to build a graphene field effect transistors dedicated to mercury detection [15]. Exploiting the formation of hydrogen bonds (H-bonds) between alkane derivative building blocks to create novel 2D structures is particularly appealing because of the strength [16] and the high selectivity and directionality of these bindings [17, 18]. It has been shown that single $[19,20,21,22,18]$ and multicomponent $[23,24,25,26,27,28,29,30,31]$ self-assembled organic nanoarchitectures can be achieved through hydrogen bonding.

Thomas et al. observed using scanning tunneling microscopy at the solid/liquid interface that the self-assembled arachidic acid layer on graphite was creating a Moiré pattern in the STM image [32]. Nath et al. also observed Moiré pattern when a 1-heptadecanol-trimesic acid layer was formed on graphite [33]. In these two cases the Moiré pattern appears like a superstructure of hexagonally ordered bright spots that are $\sim 2 \mathrm{~nm}$ large. Graphitic Moiré pattern phenomena have recently been the focus of intensive research interest because of their specific electronic properties [34, 35, 36, 37]. This type of Moiré has been exploited as twodimensional templates for creating ordered, large-scale assembly of molecules [35, 38, 39] and metal clusters [40]. In addition it has been observed that patterned adsorption of atomic hydrogen onto graphitic Moiré superlattice positions could modify honeycomb carbon layer electronic properties [39]. The ability to tailor the extremely sensitive carbon layer electronic properties through the formation of specific Moiré patterns is expected to lead to major developments in various applications, as ultra-sensitive strain gauges, pressure sensors or ultra-thin capacitors.

In this paper we investigate the self-assembly of 1-Octanol molecules $\left(\mathrm{CH}_{3}\left(\mathrm{CH}_{2}\right)_{7} \mathrm{OH}\right)$ on graphite at the solid/liquid interface. Scanning tunneling microscopy (STM) shows that 
the molecules form a two-dimensional close-packed hydrogen-bonded chevron arrangement on the surface. STM images also reveal that a Moiré pattern appears when the 1-Octanol layer is covering the graphite surface.

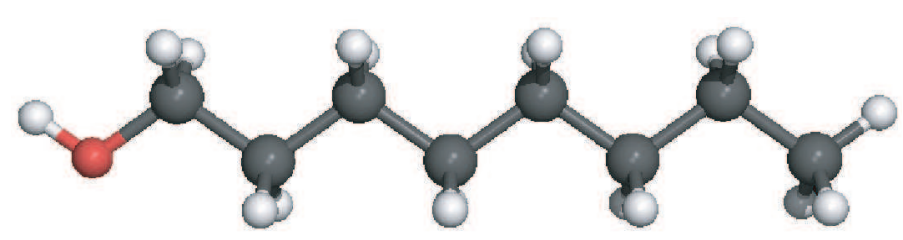

Figure 1. Scheme of 1-Octanol molecule $\left(\mathrm{CH}_{3}\left(\mathrm{CH}_{2}\right)_{7} \mathrm{OH}\right)$. Carbon atoms are gray, hydrogen atoms white and oxygen atom is red, respectively.

\section{Experimental}

A droplet of 1-Octanol solution (98\%, Acros) was deposited on a freshly cleaved graphite substrate. The droplet spread and formed a thin liquid film on the surface. The STM imaging of the samples was performed at the liquid/solid interface $1 \mathrm{~h}$ after droplet deposition using a Pico-SPM (Molecular Imaging, Agilent Technology) scanning tunneling microscope. The surface appeared to be wet during STM acquisition. Cut Pt/Ir tips were used to obtain constant current images at room temperature with a bias voltage applied to the sample. STM images were processed and analyzed using the application FabViewer [41].

\section{Results and Discussion}

The chemical structure of 1-Octanol molecule $\left(\mathrm{CH}_{3}\left(\mathrm{CH}_{2}\right)_{7} \mathrm{OH}\right)$ is presented in Figure 1. This molecule is a straight chain fatty alcohol with eight carbon atoms. This linear molecule has a hydroxyl functional group (-OH) as head and an alkyl chain as tail. The (-OH) group is expected to give a notable polar effect to the molecule.

The large scale STM image in Figure 2a reveals 1-Octanol forms large 2D nanoarchitectures at the liquid/graphite interface. STM images show that molecules selfassemble into a close-packed network. This structure is composed of organic lamellae, which are $\sim 2.1 \mathrm{~nm}$ large, Figure $2 \mathrm{~b}$. These lamellae are aligned in the $<1000\rangle$ graphite crystallographic direction. Large periodic bright stripes are visible in the STM images, Figure 2. These stripes are almost perpendicular to the molecular lamellae, i.e. they are aligned in the $<2100>$ graphite crystallographic direction. The stripe periodicity is $\sim 3.9 \mathrm{~nm}$. This domain is coexisting on the surface with similar domains rotated by an angle of $60^{\circ}$.

A high resolution STM image of the 1-Octanol network is presented in Figure 3. Molecules appear flat-lying on the surface and densely packed. The image shows that the molecules are forming a herringbone structure. The angle between the molecules in the lamella and at the lamella boundary is $120^{\circ}$. Blue arrows in Figure 3a indicate a tip change during STM image recording, revealing that STM tip apex has been modified during the scan. STM tip apex modification is a powerful method used to enhance electronic contrast in 


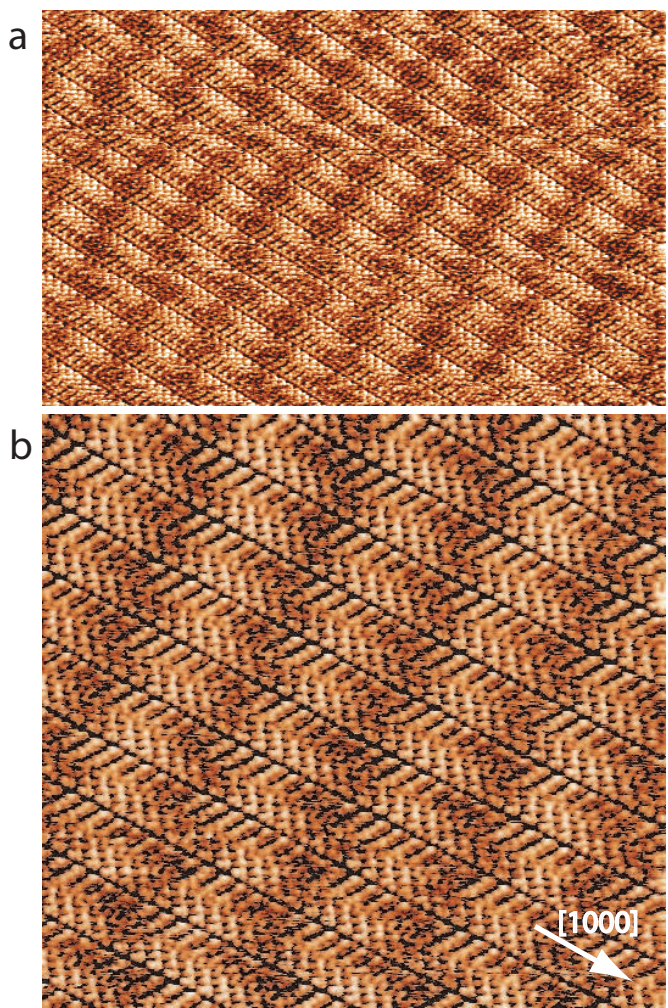

Figure 2. STM image of 1-Octanol self-assembled structures on graphite (a) $35 \times 22 \mathrm{~nm}^{2} ; \mathrm{V}_{s}=$ $0.8 \mathrm{~V}, \mathrm{I}_{t}=14 \mathrm{pA}$, (b) $17 \times 17 \mathrm{~nm}^{2} ; \mathrm{V}_{s}=0.8 \mathrm{~V}, \mathrm{I}_{t}=14 \mathrm{pA}$. The [1000] graphite crystallographic direction is indicated by a white arrow.

STM images of nanostructured surfaces [42, 43, 44, 45, 46]. It is usually used to distinguish electronic contribution from surface morphology contribution in the STM image contrast. In Figure 3a STM tip modification reveals that the integrated density of state in the center of the lamella is different from the lamella boundary, i.e. lamella center appears bright whereas lamella boundary appears dark.

We attribute this enhanced brightness in lamella center to result from hydrogen bonding between molecular hydroxyl functional group. This shows that molecules are preferentially arranged in head-to-head and tail-to-tail sequence in the $\langle 2100\rangle$ direction because a headto-tail arrangement would lead to similar density of state in the center and at the border of the lamella. The model representing the herringbone arrangement is shown in Figure $3 \mathrm{~b}$. In this model the 1-Octanol arrangement is stabilized by double $\mathrm{O} \cdots \mathrm{H}-\mathrm{O}$ hydrogen bonds between molecules; i.e. each molecule is connected to two neighbor ones through hydrogen bonds. These bonds are represented by dotted red lines in Figure $3 b$. The network unit cell is rectangular with $\sim 5.6 \AA$ and $\sim 20.0 \AA$ unit cell parameters. The molecular zigzag arrangement is the result of a flat orientation of the molecules caused by a series of $\mathrm{CH}-\pi$ interactions between the $\mathrm{CH}$ group of the alkyl chain and the $\pi$ electron system of the graphite carbon surface [47].

Figure 4 shows an large scale STM image of 1-Octanol organic layer on graphite. In the right and bottom side of the Figure, green and red rectangles have been superimposed 


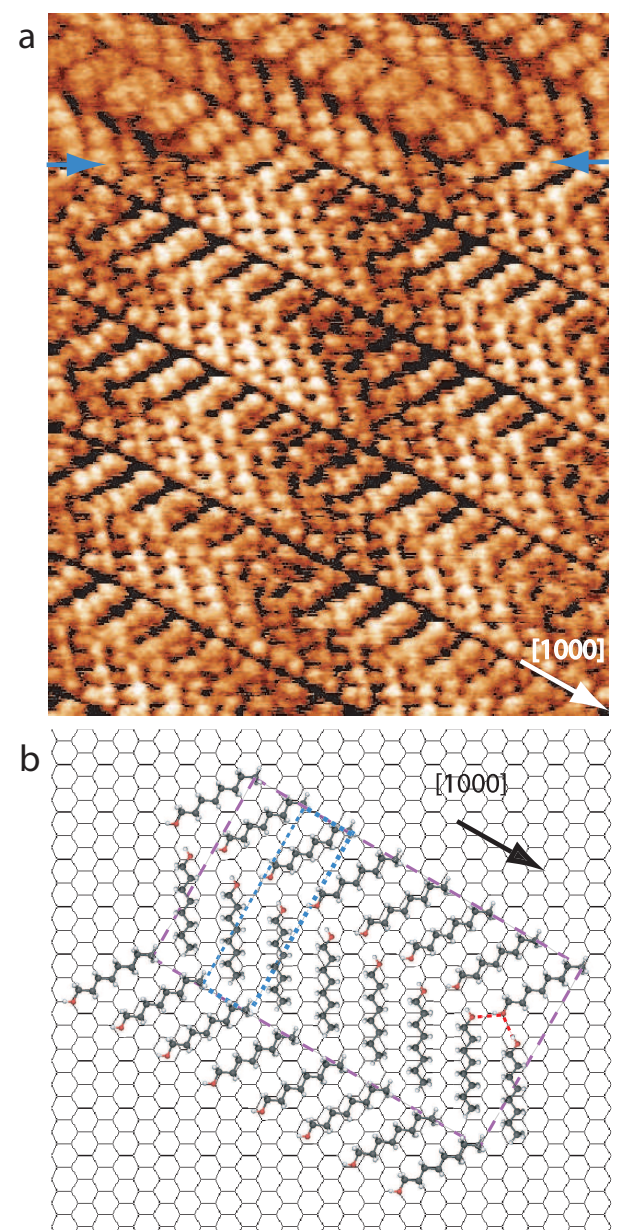

Figure 3. (a) High resolution STM image of 1 -octanol structure, $10 \times 8 \mathrm{~nm}^{2} ; \mathrm{V}_{s}=0.78 \mathrm{~V}$, $\mathrm{I}_{t}=14 \mathrm{pA}$, (b) Model of the 1-Octanol network. The network unit cell is represented by the dashed blue rectangle. Red dotted lines represent molecular hydrogen bond, the blue dotted rectangle represents the organic layer unit cell and the purple dotted rectangle represents the Moiré unit cell.

to the STM image as a guide for the eyes indicating the orientation of molecules in each network row. This image reveals that the angle between molecules of neighboring rows is generally $120^{\circ}$, except at the row boundary indicted by the black arrow in Figure 4 . At this boundary molecules are aligned in the same direction. This means that molecules are only locally arranged in a head-to-tail configuration.

STM images are showing that 1-Octanol molecules are self-assembly at the solid/liquid interface into a well-ordered organic monolayer, with molecules lying flat on the graphite surface. The organic monolayer consists in rows that are stabilized via side-by-side van der Waals interactions between the alkyl chains. STM images are showing that the image contrast in the 1-Octanol lamella center and at the lamella boundary is different. This therefore reveals that the molecules are not arranged in a head-to-tail fashion, which would lead to similar contrast. They are instead preferentially arranged alternatively in a $120^{\circ}$ head-to-head and $120^{\circ}$ tail-to-tail fashion as represented in the model Figure $3 \mathrm{~b}$. The head-to-tail arrangement was only locally observed at 1-Octanol domain boundary when molecule of neighboring 


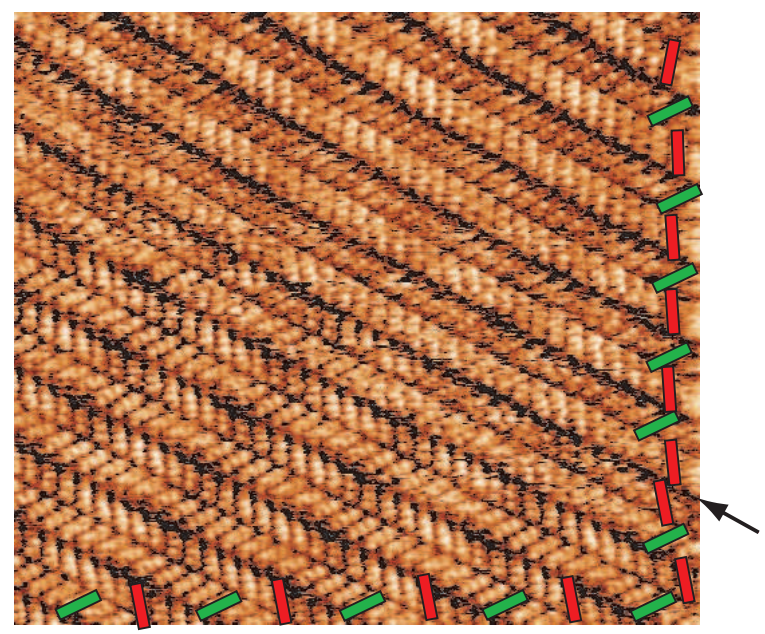

Figure 4. STM image of 1-Octanol herringbone network boundary, $15 \times 15 \mathrm{~nm}^{2} ; \mathrm{V}_{s}=0.78 \mathrm{~V}$, $\mathrm{I}_{t}=14 \mathrm{pA}$. Green (red) blocks are indicating the orientation of the molecule in each organic rows, respectively. The black arrow indicated the boundary separating two organic rows where molecules are oriented in the same direction.

rows are aligned in the same direction (Figure 4). The head-to-head structure is stabilized by double hydrogen-bonds $(2 \times \mathrm{O} \cdots \mathrm{H}-\mathrm{O})$ between molecular hydroxyl functional groups. $a b$ initio calculations showed that this $120^{\circ}$ binding leads to the formation of the most stable hydrogen-bonded arrangement in the case of long chain alcohols adsorbed on graphite or graphene surface [7]. However this structure differs from the self-assembled lamellar chevron network of 1-Octadecanethiol [43], where molecules of neighboring lamellae are aligned at the lamellar boundary (aligned tail-to-tail binding) whereas the angle is preferentially $120^{\circ}$ in the case of 1-Octanol, as observed in Figure 3 and Figure 4.

STM images also reveal that a Moiré pattern appears when the 1-Octanol herringbone network is formed on the graphite surface. In the case of 1-Octanol on graphite, the Moire pattern consists in stripes aligned in the $<2100>$ direction, perpendicular to the lamella direction. The Moiré pattern periodicity in the $<1000>$ direction is $\sim 3.9 \mathrm{~nm}$. STM images show that this distance corresponds to 71 -Octanol unit cell parameter in the $<1000>$ direction. Graphite unit cell periodicity in the $<1000>$ direction is $2.46 \AA$. We will now discuss how the superposition of two layers having different periodicities can affect STM image contrast. Moiré pattern formation results from the superposition of two lattices having different unit cell parameters. An algebraic approach can be used to calculate the points of coincidence for these two lattices. The aim is to find a structural model for the 1-Octanol adlayer and the graphite surface which is consistent with the distances and the translational symmetry seen in the STM images. The condition of coincidence of the two superposed lattices is governed by $\left(\mathrm{n} \times \lambda_{a d}\right)=(\mathrm{n} \pm 1) \times \lambda_{\text {sub }}=\lambda_{\text {Moire }}$, where $\lambda_{\text {sub }}, \lambda_{a d}$ and $\lambda_{\text {Moire }}$ denote the periodicity of the substrate surface, the 1-Octanol adsorbed layer and the Moire pattern in the $<1000>$ direction. STM images show that $\lambda_{\text {Moire }} \simeq 3.9 \mathrm{~nm}, \lambda_{a d} \simeq 5.6 \AA$ and $\mathrm{n}=7$. This leads to $\lambda_{\text {sub }} \simeq 6.5 \AA$ (considering $\mathrm{n}-1=6$ ) or $\lambda_{\text {sub }} \simeq 4.88 \AA$ (considering $\mathrm{n}+1=8$ ). These distances are not in agreement with the graphite unit cell periodicity in the $<1000>$ 
direction which is $2.461 \AA$. However it should be noticed that the Moiré periodicity would fit a substrate periodicity that verifies $39 \AA / 8=4.88 \AA$. This value is very close to the double periodicity graphite in the $<1000>$ direction that is $4.92 \AA$.

Graphite surface has been widely investigated using STM. It is well known that so-called atomically resolved STM images of graphite do not match the expected honeycomb structure of the material. There is still controversy about interpretation of the STM image of this surface [48]. It is generally admitted that STM images of graphite at the atomic scale display a hexagonal lattice where only half of the atoms are resolved. This is attributed to electronic effects $[48,49,50,51]$ because surface neighboring carbon atoms are siting in non-equivalent sites. One type of atom is sitting directly above carbon atom of the underlying layer, whereas the second type of atom is located above the center of carbon hexagon of the underlying layer. This results in the variation of the density of state of the graphite top layer due to interlayer electronic coupling. The structure observed using STM corresponds to a hexagonal network, whose periodicity is twice the one of graphite (4.92 $\AA$ ). It therefore appears that the Moiré pattern observed in the STM images presented in Figure 2 and Figure 3 corresponds to the point coincidence between the 1-Octanol layer and the electronic periodicity of the graphite surface in the $<1000>$ direction. This suggest that the contrast in the STM image corresponding to the Moiré pattern has an electronic origin resulting from the coupling of the 1-Octanol layer with the graphite surface. This effect has been mentioned by Ilan et al., who calculated that electronic coupling between alkane and graphite surface could drastically affect the appearance of individual alkane molecules in STM images [52]. Our experimental observations show that STM tip modification can drastically enhance the amplitude of the Moiré structure in the STM image, as seen in Figure 3. This is also supporting that this Moiré structure results from electronic coupling between the organic layer and the graphite surface. New theoretical development and calculations are required to assess the interface interaction and the electronic coupling between the 1-Octanol layer and the graphite surface.

\section{Conclusion}

In conclusion, scanning tunneling microscopy showed that 1-Octanol molecules selfassembled into a herringbone structure on graphite at the liquid/solid interface. This organic architecture is stabilized by hydrogen bonds. STM images also reveal that a Moiré pattern appears when the 1-Octanol herringbone network is formed onto the graphite surface. The Moiré periodicity corresponds to the point coincidence between the organic layer adlayer and twice the periodicity of the graphite surface, which corresponds to the electronic periodicity of the graphite surface. This suggests that the Moiré pattern has an electronic origin. This technique opens new opportunities to tailor the structure of graphite and graphene surfaces using layers of functionalized alkane chains having different length in order to modify surface electronic properties for new developments in applications, as ultra-sensitive strain gauges, pressure sensors or ultra-thin capacitors. 


\section{Acknowledgments}

The research leading to these results has received funding from the European Research Council under the European Union's Seventh Framework Programme (FP7/2007-2013) / ERC grant agreement $\mathrm{n}^{\circ} 259297$.

\section{References}

[1] Hailin Liang, Wei Sun, Xin Jin, Heng Li, Jianlong Li, Xinquan Hu, Boon K Teo, and Kai Wu. TwoDimensional molecular porous networks formed by trimesic acid and 4,4'Bis(4-pyridyl)biphenyl on au(111) through hierarchical hydrogen bonds: Structural systematics and control of nanopore size and shape. Angew. Chem. Int. Edn, 50(33):7562-7566, August 2011.

[2] Yanlian Yang and Chen Wang. Hierarchical construction of self-assembled low-dimensional molecular architectures observed by using scanning tunneling microscopy. Chem. Soc. Rev., 38(9):2576, 2009.

[3] Sebastian Stepanow, Nian Lin, Dietmar Payer, Uta Schlickum, Florian Klappenberger, Giorgio Zoppellaro, Mario Ruben, Harald Brune, JohannesV. Barth, and Klaus Kern. Surface-Assisted assembly of 2D MetalOrganic networks that exhibit unusual threefold coordination symmetry. Angew. Chem. Int. Edn, 46(5):710-713, January 2007.

[4] D. S Deak, F. Silly, K. Porfyrakis, and M. R Castell. Controlled surface ordering of endohedral fullerenes with a SrTiO3 template. Nanotechnology, 18:075301, 2007.

[5] Chao Lu, Erkuang Zhu, Yadi Liu, Zhongyuan Liu, Yafeng Lu, Julong He, Dongli Yu, Yongjun Tian, and Bo Xu. C60 on nanostructured Nb-Doped SrTiO3(001) surfaces. J. Phys. Chem. C, 114(8):3416-3421, March 2010.

[6] Yung-Fang Liu and Yuh-Lang Lee. Adsorption characteristics of OH-terminated alkanethiol and arenethiol on au(111) surfaces. Nanoscale, 4(6):2093-2100, March 2012.

[7] Teng Yang, Savas Berber, Jun-Fu Liu, Glen P Miller, and David Tomanek. Self-assembly of long chain alkanes and their derivatives on graphite. J. Chem. Phys., 128(12):124709-124709-8, March 2008.

[8] O. Marchenko and J. Cousty. Molecule length-induced reentrant self-organization of alkanes in monolayers adsorbed on au (111). Phys. Rev. Lett., 84(23):5363-5366, 2000.

[9] Thomas Müller, Tova L Werblowsky, Gina M Florio, Bruce J Berne, and George W Flynn. Ultra-high vacuum scanning tunneling microscopy and theoretical studies of 1-halohexane monolayers on graphite. P. Natl. Acad. Sci. USA, 102(15):5315-5322, April 2005.

[10] Jrgen P Rabe and Stefan Buchholz. Commensurability and mobility in Two-Dimensional molecular patterns on graphite. Science, 253(5018):424-427, July 1991.

[11] Thomas Müller, Tova L Werblowsky, Gina M Florio, Bruce J Berne, and George W Flynn. Ultra-High vacuum scanning tunneling microscopy and theoretical studies of 1-Halohexane monolayers on graphite. P. Natl. Acad. Sci. USA, 102(15):5315-5322, April 2005.

[12] Shan-Shan Li, Li-Ping Xu, Li-Jun Wan, Shu-Tao Wang, and Lei Jiang. Time-Dependent organization and wettability of decanethiol Self-Assembled monolayer on au(111) investigated with STM. J. Phys. Chem. $B, 110(4): 1794-1799,2006$.

[13] Osamu Endo, Hiroyuki Ozaki, Ryouhei Sumii, Kenta Amemiya, Masashi Nakamura, and Nobuhiro Kosugi. Orientation of n-alkane in thin films on graphite $\left(\begin{array}{llll}0 & 0 & 0 & 1\end{array}\right)$ studied using c K-NEXAFS. J. Electron Spectrosc., 184(3-6):257-260, April 2011.

[14] Feng Tao, Julie Goswami, and Steven L. Bernasek. Self-Assembly and Odd-Even effects of cisUnsaturated carboxylic acids on highly oriented pyrolytic graphite. J. Phys. Chem. B, 110(9):41994206, 2006.

[15] Tao Zhang, Zengguang Cheng, Yibing Wang, Zhongjun Li, Chenxuan Wang, Yibao Li, and Ying Fang. Self-Assembled 1-Octadecanethiol monolayers on graphene for mercury detection. Nano Lett., 10(11):4738-4741, 2010. 
[16] Johannes V. Barth. Molecular architectonic on metal surfaces. Ann. Rev. Phys. Chem., 58(1):375-407, May 2007.

[17] Prabhpreet Singh, V. Venkatesh, N. Nagapradeep, Sandeep Verma, and Alberto Bianco. G-quartet type self-assembly of guanine functionalized single-walled carbon nanotubes. Nanoscale, 4(6):1972-1974, March 2012.

[18] M. Mura, F. Silly, G. A. D. Briggs, M. R. Castell, and L. N. Kantorovich. H-Bonding supramolecular assemblies of PTCDI molecules on the au(111) surface. J. Phys. Chem. C, 113(52):21840-21848, December 2009.

[19] Bulent Baris, Vincent Luzet, Eric Duverger, Philippe Sonnet, Frank Palmino, and Frederic Cherioux. Robust and open tailored supramolecular networks controlled by the template effect of a silicon surface. Angew. Chem. Int. Edn, 50(18):4094-4098, April 2011.

[20] M. Mura, X. Sun, F. Silly, H. T. Jonkman, G. A. D. Briggs, M. R. Castell, and L. N. Kantorovich. Experimental and theoretical analysis of h-bonded supramolecular assemblies of PTCDA molecules. Phys. Rev. B, 81(19):195412, May 2010.

[21] Shinobu Uemura, Masashi Aono, Tamikuni Komatsu, and Masashi Kunitake. Two-Dimensional SelfAssembled structures of melamine and melem at the aqueous SolutionAu(111) interface. Langmuir, 27(4):1336-1340, February 2011.

[22] Qing Hua Wang and Mark C. Hersam. Room-temperature molecular-resolution characterization of selfassembled organic monolayers on epitaxial graphene. Nature Chemistry, 1(3):206-211, May 2009.

[23] Fabien Silly, Adam Q. Shaw, G. A. D. Briggs, and Martin R. Castell. Epitaxial ordering of a perylenetetracarboxylic diimide-melamine supramolecular network driven by the au(111)-(223) reconstruction. Appl. Phys. Lett., 92(2):023102, 2008.

[24] Xiaobo Mao, Chenxuan Wang, Xiaojing Ma, Min Zhang, Lei Liu, Lan Zhang, Lin Niu, Qindao Zeng, Yanlian Yang, and Chen Wang. Molecular level studies on binding modes of labeling molecules with polyalanine peptides. Nanoscale, 3(4):1592-1599, April 2011.

[25] Li-Ping Xu, Yibiao Liu, and Xueji Zhang. Interfacial self-assembly of amino acids and peptides: Scanning tunneling microscopy investigation. Nanoscale, 3(12):4901-4915, November 2011.

[26] Xiaonan Sun, Manuela Mura, Harry T. Jonkman, Lev N. Kantorovich, and Fabien Silly. Fabrication of a complex Two-Dimensional Adenine-Perylene-3,4,9,10-tetracarboxylic dianhydride chiral nanoarchitecture through molecular Self-Assembly. J. Phys. Chem. C, 116(3):2493-2499, 2012.

[27] S. Jensen, J. Greenwood, H. A. Fruchtl, and C. J. Baddeley. STM investigation on the formation of oligoamides on au111 by Surface-Confined reactions of melamine with trimesoyl chloride. J. Phys. Chem. C, 115(17):8630-8636, May 2011.

[28] Yibao Li, Keqing Zhao, Yanlian Yang, Ke Deng, Qingdao Zeng, and Chen Wang. Functionalization of two-component molecular networks: recognition of $\mathrm{fe}^{3+}$. Nanoscale, 4(1):148-151, December 2011.

[29] Xiaonan Sun, Harry T Jonkman, and Fabien Silly. Tailoring two-dimensional PTCDA-melamine self-assembled architectures at room temperature by tuning molecular ratio. Nanotechnology, 21(16):165602, April 2010.

[30] Jules A. Gardener, Olga Y. Shvarova, G. Andrew D. Briggs, and Martin R. Castell. Intricate HydrogenBonded networks: Binary and ternary combinations of uracil, PTCDI, and melamine. J. Phys. Chem. C, 114(13):5859-5866, April 2010.

[31] Chenxuan Wang, Aihua Yang, Xia Li, Denghua Li, Min Zhang, Huiwen Du, Chao Li, Yuanyuan Guo, Xiaobo Mao, Mingdong Dong, Flemming Besenbacher, Yanlian Yang, and Chen Wang. Observation of molecular inhibition and binding structures of amyloid peptides. Nanoscale, 4(6):1895-1909, March 2012.

[32] Loji K. Thomas, Angelika Kuhnle, Sebastian Rode, Uwe Beginn, and Michael Reichling. Monolayer structure of arachidic acid on graphite. J. Phys. Chem. C, 114(44):18919-18924, 2010.

[33] Krishna G. Nath, Oleksandr Ivasenko, Jennifer M. MacLeod, Jill A. Miwa, James D. Wuest, Antonio Nanci, Dmitrii F. Perepichka, and Federico Rosei. Crystal engineering in two dimensions: an approach to molecular nanopatterning. J. Phys. Chem. C, 111(45):16996-17007, 2007.

[34] I. Pletikosi, M. Kralj, P. Pervan, R. Brako, J. Coraux, A. T. NDiaye, C. Busse, and T. Michely. Dirac cones 
and minigaps for graphene on ir(111). Phys. Rev. Lett., 102(5):056808, February 2009.

[35] H. G. Zhang, J. T. Sun, T. Low, L. Z. Zhang, Y. Pan, Q. Liu, J. H. Mao, H. T. Zhou, H. M. Guo, S. X. Du, F. Guinea, and H.-J. Gao. Assembly of iron phthalocyanine and pentacene molecules on a graphene monolayer grown on ru(0001). Phys. Rev. B, 84(24):245436, December 2011.

[36] A. Luican, Guohong Li, A. Reina, J. Kong, R. R. Nair, K. S. Novoselov, A. K. Geim, and E. Y. Andrei. Single-Layer behavior and its breakdown in twisted graphene layers. Phys. Rev. Lett., 106(12):126802, March 2011.

[37] S. Shallcross, S. Sharma, E. Kandelaki, and O. A. Pankratov. Electronic structure of turbostratic graphene. Phys. Rev. B, 81(16):165105, April 2010.

[38] Jinhai Mao, Haigang Zhang, Yuhang Jiang, Yi Pan, Min Gao, Wende Xiao, and H.-J. Gao. Tunability of supramolecular kagome lattices of magnetic phthalocyanines using Graphene-Based moire patterns as templates. J. Am. Chem. Soc., 131(40):14136-14137, October 2009.

[39] Richard Balog, Bjarke Jørgensen, Louis Nilsson, Mie Andersen, Emile Rienks, Marco Bianchi, Mattia Fanetti, Erik Lægsgaard, Alessandro Baraldi, Silvano Lizzit, Zeljko Sljivancanin, Flemming Besenbacher, Bjørk Hammer, Thomas G. Pedersen, Philip Hofmann, and Liv Hornekær. Bandgap opening in graphene induced by patterned hydrogen adsorption. Nature Mater., 9(4):315-319, March 2010.

[40] Yi Pan, Min Gao, Li Huang, Feng Liu, and H.-J. Gao. Directed self-assembly of monodispersed platinum nanoclusters on graphene moire template. Appl. Phys. Lett., 95(9):093106, 2009.

[41] F. Silly. A robust method for processing scanning probe microscopy images and determining nanoobject position and dimensions. . Microsc.-Oxf., 236(3):211-218, 2009.

[42] Martin R. Castell. Scanning tunneling microscopy of reconstructions on the $\mathrm{srtio}_{3}(001)$ surface. Surf. Sci., 505:1-13, May 2002.

[43] Qing-Min Xu, Li-Jun Wan, Shu-Xia Yin, Chen Wang, and Chun-Li Bai. Effect of chemically modified tips on STM imaging of 1-Octadecanethiol molecule. J. Phys. Chem. B, 105(43):10465-10467, 2001.

[44] R. Temirov, S. Soubatch, O. Neucheva, A. C. Lassise, and F. S. Tautz. A novel method achieving ultra-high geometrical resolution in scanning tunnelling microscopy. N. J. Phys., 10:053012, 2008.

[45] H S Wong, X Feng, K Müllen, N Chandrasekhar, and C Durkan. Channel selective tunnelling through a nanographene assembly. Nanotechnology, 23(9):095601, March 2012.

[46] B.C. Russell and M.R. Castell. Surface of sputtered and annealed polar SrTiO3(111): TiOx-Rich (n n) reconstructions. J. Phys. Chem. C, 112(16):6538-6545, April 2008.

[47] Lluïsa Pérez-Garcia and David B. Amabilino. Spontaneous resolution under supramolecular control. Chem. Soc. Rev., 31(6):342-356, October 2002.

[48] F. Atamny, O. Spillecke, and R. Schlögl. On the STM imaging contrast of graphite: towards a true atomic resolution. Phys. Chem. Chem. Phys., 1(17):4113-4118, January 1999.

[49] David Tomnek, Steven G. Louie, H. Jonathon Mamin, David W. Abraham, Ruth Ellen Thomson, Eric Ganz, and John Clarke. Theory and observation of highly asymmetric atomic structure in scanningtunneling-microscopy images of graphite. Phys. Rev. B, 35(14):7790-7793, May 1987.

[50] Inder P. Batra, N. Garca, H. Rohrer, H. Salemink, E. Stoll, and S. Ciraci. A study of graphite surface with stm and electronic structure calculations. Surf. Sci., 181(12):126-138, March 1987.

[51] Kee Hag Lee, M. Caus, and Sung Soo Park. Ab initio periodic HartreeFock calculations for interpretation of the scanning tunneling microscope (STM) images of graphite. J. Phys. Chem. B, 102(31):6020-6024, 1998.

[52] Boaz Ilan, Gina M. Florio, Mark S. Hybertsen, B. J. Berne, and George W. Flynn. Scanning tunneling microscopy images of alkane derivatives on graphite: Role of electronic effects. Nano Lett., 8(10):3160$3165,2008$. 